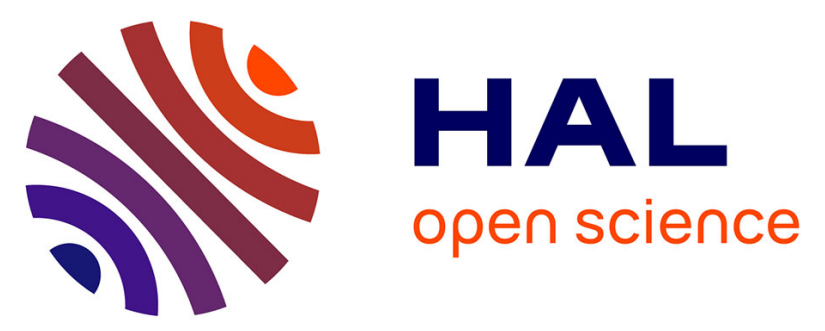

\title{
Etude comparative des migrations d'assimilats chez deux variétés de soja, de type déterminé ou indéterminé. Influence d'une carence en azote
}

Jacqueline Grima Pettenati, Geneviève Bailly-Fenech, Jean-Claude Latché

\section{- To cite this version:}

Jacqueline Grima Pettenati, Geneviève Bailly-Fenech, Jean-Claude Latché. Etude comparative des migrations d'assimilats chez deux variétés de soja, de type déterminé ou indéterminé. Influence d'une carence en azote. Agronomie, 1987, 7 (6), pp.447-456. hal-00885014

\section{HAL Id: hal-00885014 \\ https://hal.science/hal-00885014}

Submitted on 1 Jan 1987

HAL is a multi-disciplinary open access archive for the deposit and dissemination of scientific research documents, whether they are published or not. The documents may come from teaching and research institutions in France or abroad, or from public or private research centers.
L'archive ouverte pluridisciplinaire HAL, est destinée au dépôt et à la diffusion de documents scientifiques de niveau recherche, publiés ou non, émanant des établissements d'enseignement et de recherche français ou étrangers, des laboratoires publics ou privés. 


\title{
Etude comparative des migrations d'assimilats chez deux variétés de soja, de type déterminé ou indéterminé. Influence d'une carence en azote
}

\author{
Jacqueline GRIMA-PETTENATI, Geneviève BAILLY-FENECH et Jean-Claude LATCHÉ \\ Centre de Physiologie végétale, Unité associée au C.N.R.S. $n^{\circ} 241$, Université Paul Sabatier, 118 , route de \\ Narbonne, 31062 Toulouse Cedex
}

RÉSUMÉ

\begin{abstract}
Après fixation photosynthétique de ${ }^{14} \mathrm{CO}_{2}$ par une feuille de niveau moyen, la migration des composés élaborés a été suivie, à divers stades de développement, chez les variétés de Soja M 13, de type déterminé, et Amsoy 71, de type indéterminé. Dans les plantes jeunes, les parties végétatives en cours de croissance constituent les principaux organes receveurs ; les fructifications présentent ensuite les indices de marquage spécifique les plus élevés, quoique variables selon l'âge physiologique des gousses. La variété M 13 se caractérise par une meilleure exportation des assimilats vers les étages inférieurs, et en particulier vers les ramifications. Pour la variété Amsoy 71 , les transports vers les organes apicaux se poursuivent plus longtemps durant le cycle de développement ; dans les deux cas, on note une orientation longitudinale nettement préférentielle des migrations et une absence d'échanges entre feuilles adultes. Les formes de transport diffèrent selon l'âge de la plante mais le saccharose et l'asparagine jouent toujours un rôle primordial. Une carence en azote, réalisée à partir de la floraison, entraîne une réduction à la fois de la vitesse et du volume des échanges; en outre, les exportations vers les racines sont accrues.
\end{abstract}

Mots clés additionnels : Glycine max (L.) Merr. cv M 13 et Amsoy 7l, fixation photosynthétique de ${ }^{14} \mathrm{CO}_{2}$, indices de marquage spécifique, formes de transport.

Photosynthate translocation in soybean : comparison between determinate and indeterminate types of development and effects of nitrogen starvation.

After photosynthetic ${ }^{14} \mathrm{CO}_{2}$ assimilation by a leaf from the medium part of the canopy, the distribution of labelled compounds was examined, at various developmental stages, in soybean cultivars M 13 (determinate type) and Amsoy 71 (indeterminate type). Developing vegetative organs acted as major sinks in young plants ; the highest relative specific activities were then observed in pods, but they varied with the growth stage of the fruits. Cultivar M 13 differed by better assimilate export towards basal plant parts and particularly to the secondary axes. In cultivar Amsoy 71, assimilate movement towards the shoot apex remained effective during the pod-filling period. In both genotypes, photosynthate migrated predominantly to organs located at the same side as the source leaf and there was little, or no, carbon transfer between mature leaves. The nature of the translocated compounds varied with the age of the soybean plant, but the radioactivity of sucrose and asparagine was always high in conducting tissues. Nitrogen starvation of plants from flowering promoted a decrease in both rate and amount of assimilate translocation ; moreover, the export of organic compounds towards the roots was increased.

Additional key words : Glycine max (L.) Merr. cv M 13 and Amsoy 71, photosynthetic fixation of ${ }^{14} \mathrm{CO}_{2}$, relative specific activities, translocated compounds.

\section{INTRODUCTION}

Le développement des organes fructifères chez le soja est étroitement lié au transfert des assimilats élaborés dans le limbe foliaire (STEPHENSON \& WILSON, 1977 ; BONNEMAIN, 1978). Les gousses ne présentent en effet qu'une faible capacité de fixation photosyn- thétique du gaz carbonique (ANDREwS \& SVEC, 1975 ; LATCHÉ et al., 1986). En outre, leur activité photorespiratoire est élevée (LATCHE et al., 1986), ce qui contribue à abaisser le taux de photosynthèse nette et, selon SAMBO et al. (1977), elles ne peuvent satisfaire au maximum que 4 p. 100 de leurs propres besoins en carbone. 
Divers auteurs ont établi que la migration des photosynthétats était en partie contrôlée par les conditions de culture. Ainsi interviennent l'intensité de l'éclairement reçu par la plante (HEINDL \& BRUN, 1983), la durée de l'héméropériode (CURE et al., 1982), la valeur de la température nocturne (SEDDIGH \& JOLIFF, 1984), l'approvisionnement en eau (HUBER et al. $1984 b$; CALMÉS et al., 1985), la teneur en $\mathrm{CO}_{2}$ de l'atmosphère (HUBER et al., 1984a) ou la concentration en potassium du milieu (HUBER, 1984).

Le présent travail se propose de préciser les éventuelles répercussions, sur les transferts de composés organiques, d'une privation d'azote. Deux raisons justifient le choix de ce paramètre. Etant donné la richesse en protéines des graines de soja (40 p. 100 de la masse de matière sèche), la nutrition azotée est une composante importante du rendement. Par ailleurs, dans ses conditions naturelles de culture, le soja peut se trouver en situation de carence partielle en azote. Ainsi, en période de sécheresse, la fixation symbiotique du $\mathrm{N}_{2}$ est fortement diminuée (OBATON, 1980); l'assimilation des ions nitrate du sol ne compense pas ce déficit, en particulier au cours de la phase de fructification durant laquelle la plante ne présente qu'une activité nitrate réductase limitée (OBATON, 1980).

Le comportement de deux variétés, à croissance de type déterminé ou indéterminé, a été suivi comparativement. Après une étude de l'exportation des assimilats à partir d'une feuille de niveau moyen chez des plantes normalement alimentées, les effets d'une carence en azote ont été examinés.

\section{MATÉRIEL ET MÉTHODES}

\section{A. Matériel végétal}

Les cultivars M 13 (type déterminé) et Amsoy 71 (type indéterminé), d'origine américaine et de groupe de précocité II, ont été retenus comme matériel végétal. Ils sont cultivés en conditions contrôlées (éclairement : $330 \mathrm{~mol} \cdot \mathrm{m}^{-2} \cdot \mathrm{s}^{-1}$; héméropériode : $14 \mathrm{~h}$; température : $25^{\circ} \mathrm{C}$ en phase éclairée et $18^{\circ} \mathrm{C}$ en phase obscure ; hygrométrie moyenne : 80 p. 100 de la saturation), sur solutions synthétiques liquides (BouNIOLs et al., 1981). Les plantes, non nodulées, sont d'abord alimentées par un milieu complet $(\mathrm{N}+)$ dans lequel l'azote est apporté essentiellement sous forme nitrique. En début de floraison, deux lots sont constitués : le premier est maintenu sur milieu $\mathrm{N}+$, le second est transféré sur une solution dépourvue de source azotée (No).

Comparativement à des cultures réalisées au champ, le cycle de végétation en conditions contrôlées est considérablement raccourci : les premières fleurs apparaissent en moyenne 35 à 38 jours après le semis, le grossissement des graines débutant 12 à 15 jours plus tard. Cette accélération du développement serait due surtout, selon OLIKER et al. (1978), à la valeur élevée de la température nocturne. Quatre étapes ont été choisies pour expérimentation : elles se situent successivement durant la période de croissance végétative (stade I, 7 jours avant le début de la floraison), en début de floraison (stade II), et pendant la phase de grossissement des graines, soit 14 jours (stade III) el 21 jours (stade IV) après la mise à fleurs ; les stades II, III et IV correspondent respectivement aux stades $R_{2}$, $\mathrm{R}_{4}$ et $\mathrm{R}_{6}$ définis par FEHR et al. (1971).

Les feuilles trifoliolées (F) portées par la tige principale sont numérotées selon leur ordre d'apparition.

\section{B. Techniques expérimentales}

La fixation de ${ }^{14} \mathrm{CO}_{2}$ par une feuille adulte d'étage médian, soit d'une part $F_{3}$ (stade I) et $F_{5}$ (stades II, III et IV) pour la variété $M 13$, d'autre part $F_{3}$ (stade I), $F_{5}$ (stade II) et $F_{8}$ (stades III et IV) pour la variétć Amsoy 71, permet de marquer in situ les photosynthétats. Le limbe est introduit dans une enceinte éclairée $\left(330 \mu \mathrm{mol} . \mathrm{m}^{-2} . \mathrm{s}^{-1}\right)$ et, après balayage (15 $\mathrm{mn}$ ) par un courant d'air dépourvu de gaz carbonique afin de favoriser l'ouverture des stomates (LOUGUET, 1978), il est mis en contact de ${ }^{14} \mathrm{CO}_{2}$ (400 vpm ; $370 \mathrm{KBq}$ ) obtenu par addition d'acide lactique à une solution de $\mathrm{NaH}^{14} \mathrm{CO}_{3}$. La durée de l'assimilation est fixée à $40 \mathrm{mn}$. La plante est ensuite transférée sous une hotte ventilée (température : 20 à $22^{\circ} \mathrm{C}$ ), ou pendant $6 \mathrm{~h}$ sous un éclairement de $260 \mu \mathrm{mol} . \mathrm{m}^{-2} . \mathrm{s}^{-1}$, ou durant $24 \mathrm{~h}$ à raison de $14 \mathrm{~h}$ d'éclairement et de $10 \mathrm{~h}$ d'obscurité. Au terme de la période de chasse retenue, les différents organes du végétal sont prélevés, lyophilisés et réduits en poudre. Leur teneur globale en radiocarbone est déterminée après combustion (Oxidizer Packard, type Tricarb 306).

Le protocole expérimental adopté pour l'identification des métabolites marqués est analogue à celui décrit précédemment (KPODAR et al., 1978) ; les composés sont extraits en milieux hydroéthanoliques, séparés par chromatographie de partage sur papier et repérés par autoradiographie (film Kodak X-Omat S).

Les mesures de radioactivité sont réalisées par scintillation en milieu liquide (mélange scintillant Unisolve), à l'aide d'un spectromètre Packard, type CSL $460 \mathrm{C}$.

\section{RÉSULTATS ET DISCUSSION}

\section{A. Etude des migrations chez les plantes cultivées sur milieu complet}

\section{Importance des transferts d'assimilats en fonction du stade de développement}

Le transfert global des assimilats, après une période de chasse de $6 \mathrm{~h}$ (fig. 1A), ne représente jamais plus de 50 p. 100 du radiocarbone fixé par la feuille alimentée en ${ }^{14} \mathrm{CO}_{2}$; un résultat similaire a été observé par FELlows et al. (1978). En ce qui concerne Amsoy 71 , de type indéterminé, on constate que les exportations, déjà élevées au stade I, passent par un maximum aux stades II et III. Chez le cultivar M 13, le volume des migrations est nettement moins impor- 
tant durant la phase de développement végétatif et à la floraison ; il augmente en revanche de façon marquée au stade III et il se maintient sensiblement au même niveau au stade IV. Des constatations analogues ont été effectuées après $24 \mathrm{~h}$ de chasse (fig. 1B) ; il semble donc que, pour la variété de type déterminé, les feuilles soient susceptibles d'exporter une part plus grande des photosynthétats durant la phase de fort grossissement des graines.

\section{Principaux organes receveurs}

Selon BONNEMAIN (1978), chez plusieurs espèces végétales dont le soja, les feuilles de l'étage moyen fournissent des assimilats à la fois aux racines et aux organes aériens en voie de croissance. Ces derniers, comme le montre le tableau 1 , constituent effectivement les principaux « puits » dans les plantes jeunes ; toutefois, à partir de la floraison, les composés organiques sont dirigés de façon préférentielle vers les étages inférieurs ou vers les gousses insérées, sur la tige principale, à l'aisselle de la feuille alimentée en ${ }^{14} \mathrm{CO}_{2}$. De nettes différences apparaissent cependant en fonction de la variété étudiée. Chez M 13, la migration des photosynthétats vers le haut de la plante est, de façon générale, moins importante et elle diminue beaucoup plus précocément, dès le stade II ; par ailleurs, on note un meilleur approvisionnement des ramifications en composés marqués. En revanche, dans le cas d'Amsoy 71, les parties aériennes en cours de développement au sommet de la tige, les racines et les gousses portées par l'axe principal reçoivent proportionnellement davantage de radiocarbone.

Le calcul des indices de marquage spécifique (IMS) permet de préciser la nature des principaux organes receveurs. L'IMS est le rapport entre la radioactivité (exprimée en p. 100 du marquage total de la plante) et la masse de matière sèche (exprimée en p. 100 de la masse de matière sèche du végétal). Plus l'IMS est

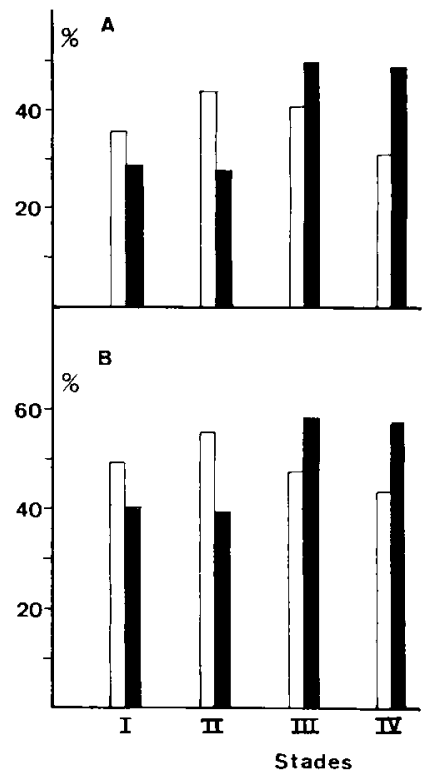

Figure 1

Radioactivité totale des métabolites exportés à partir de la feuille alimentée en ${ }^{14} \mathrm{CO}_{2}$, après une période de chasse de $6 \mathrm{~h}(\mathrm{~A})$ ou $24 h(B)$. Résultats exprimés en \% de la radioactivité fixée. $\square$ variété Amsoy $71 ; \square$ variété M 13 ; plantes cultivées sur milieu complet.

Total labelling of metabolites exported from the source leaf fed with ${ }^{14} \mathrm{CO}_{2}$ after a $6 \mathrm{~h}(\mathrm{~A})$ or $24 \mathrm{~h}(\mathrm{~B})$ chase period. Results expressed as \% of fixed ${ }^{14} C$.

$\square$ cultivar Amsoy $71 ; \square$ cultivar $M 13$; plants grown on complete medium.

élevé, plus la « force puits » de l'organe auquel il correspond est importante (BRUN \& BETTS, 1984). L'examen du tableau 2 montre, qu'au stade I, les feuilles en voie de croissance des étages supérieurs présentent de forts IMS, à la fois chez M 13 et Amsoy 71. Pour cette dernière variété, la même constatation reste valable au stade II ; en revanche, chez M 13 , les tissus

\section{TABLEAU 1}

Répartition, en \% du radiocarbone exporté à partir du limbe de la feuille alimentée en ${ }^{14} \mathrm{CO}_{2}$ dans les végétaux cultivés sur milieu complet. $\mathrm{F}_{-}{ }^{14} \mathrm{CO}_{2}$ : feuille ayant fixé le gaz carbonique marqué; $V$ : organes végétatifs ; $R$ : orgunes reproducteurs ; durée de chasse : $6 \mathrm{~h}$. Distribution, in $\%$, of ${ }^{14} \mathrm{C}$ exported from the blade of the source leaf fed with ${ }^{14} \mathrm{CO}_{2}$. Plants grown on complete medium. $\mathrm{F}_{-}{ }^{14} \mathrm{CO}_{2}$ : source leaf ; $V$ : vegetative organs ; $R$ : flowers or pods; chase period : $6 \mathrm{~h}$.

\begin{tabular}{|c|c|c|c|c|c|c|c|c|c|}
\hline & & \multicolumn{4}{|c|}{ Variété M 13} & \multicolumn{4}{|c|}{ Variété Amsoy 71} \\
\hline & & Stade I & Stade II & Stade III & Stade IV & Stade I & Stade II & Stade III & Stade IV \\
\hline Pétiole $\mathrm{F}^{14}{ }^{14} \mathrm{CO}_{2}$ & & 10,9 & 12,4 & 6,0 & 3,4 & 8,3 & 5,2 & 4,3 & 4,7 \\
\hline \multicolumn{10}{|c|}{$\begin{array}{l}\text { Organes insérés à l'aisselle de } \mathrm{F}_{-}{ }^{14} \mathrm{CO}_{2} \text { : } \\
\text { Gousses ou fleurs }\end{array}$} \\
\hline \multirow{2}{*}{ Ramifications } & $\mathrm{v}$ & $\overline{-}$ & $\underline{3,7}$ & $\begin{array}{r}24,1 \\
0,4\end{array}$ & $\begin{array}{r}28,7 \\
0.9\end{array}$ & - & $\begin{array}{l}7,9 \\
-\end{array}$ & $\begin{array}{r}17,1 \\
0,1\end{array}$ & $\begin{array}{r}41,7 \\
0,3\end{array}$ \\
\hline & $\mathbf{R}$ & - & - & 0,3 & 1,7 & - & - & 0,1 & 0,3 \\
\hline \multicolumn{10}{|c|}{ Organes niveaux supérieurs à $\mathrm{F}_{-}{ }^{14} \mathrm{CO}_{2}$ : } \\
\hline \multirow{2}{*}{ Axe principal } & $\mathrm{V}$ & 34,5 & 4,7 & 1,3 & 0,8 & 44,6 & 24,4 & 8,5 & 3,2 \\
\hline & $\mathbf{R}$ & - & - & 0,8 & 0,8 & - & 2,6 & 9,5 & 2,3 \\
\hline \multirow{2}{*}{ Ramifications } & $\mathrm{V}$ & - & - & - & 0,1 & - & - & - & 0,3 \\
\hline & $\mathbf{R}$ & - & - & - & 0,4 & - & - & - & 0,5 \\
\hline \multicolumn{10}{|c|}{ Organes niveaux inférieurs à $\mathrm{F}_{-}{ }^{14} \mathrm{CO}_{2}$ : } \\
\hline \multirow{2}{*}{ Axe principal } & $\mathrm{V}$ & 20,3 & 19,7 & 12,8 & 6,8 & 21,4 & 20,0 & 18,1 & 17,4 \\
\hline & $\mathbf{R}$ & - & 2,8 & 8,2 & 12,8 & - & 2,6 & 15,4 & 9,6 \\
\hline \multirow{2}{*}{ Ramifications } & $\mathrm{V}$ & 17,8 & 17,4 & 5,1 & 2,6 & 1,0 & 4,2 & 1,2 & 0,3 \\
\hline & $\mathbf{R}$ & - & - & 22,6 & 34,2 & - & - & 1,3 & 1,0 \\
\hline Racines & & 16,5 & 39,3 & 18,4 & 6,8 & 24,7 & 33,1 & 24,4 & 18,4 \\
\hline
\end{tabular}




\section{TABLEAU 2}

Indice de marquage spécifique (IMS $=\%$ radioactivité/\% masse à l'état sec) des différents organes, aux stades I et II. $\mathrm{F}_{-}{ }^{14} \mathrm{CO}_{2}$ : feuille ayant assimilé le gaz carbonique marqué ; durée de chasse $: 6 \mathrm{~h} ;+:$ IMS inférieur a $(0,1$.

Relative specific activity (\% labelling $\%$ dry weight) of different plant parts, at developmental stages I and II. $\mathrm{F}_{-}{ }^{14} \mathrm{CO}_{2}$ : source leaf ; chase period : $6 \mathrm{~h} ;+$ : relative specific activity $<0.1$.

\begin{tabular}{|c|c|c|c|c|}
\hline & \multicolumn{2}{|c|}{ Variété M 13} & \multicolumn{2}{|c|}{ Variété Amsoy 71} \\
\hline & Stade I & Stade II & Stade I & Stade II \\
\hline Limbe $\mathrm{F}^{-14} \mathrm{CO}_{2}$ & 15,4 & 14,6 & 12,3 & 11,9 \\
\hline Pétiole $\mathrm{F}{ }^{14} \mathrm{CO}_{2}$ & 1,9 & 3,1 & 3,1 & 3,8 \\
\hline Fleurs aisselle $\mathrm{F}_{-}{ }^{14} \mathrm{CO}_{2}$ & - & 2,3 & - & 4,6 \\
\hline \multicolumn{5}{|l|}{ Etages supérieurs à $F-{ }^{14} \mathrm{CO}_{2}$ : } \\
\hline Feuilles adultes & 0,1 & + & + & + \\
\hline Feuilles en croissance + bourgeons & 2,6 & 0,1 & 3,2 & 3,5 \\
\hline Entre-nœuds & 1,5 & 0,2 & 1,7 & 1,8 \\
\hline Fleurs & - & 0,5 & - & 3,2 \\
\hline \multicolumn{5}{|l|}{ Etages inférieurs à $\mathrm{F}^{14} \mathrm{CO}_{2}$ : } \\
\hline \multirow{3}{*}{ Axe principal } & 0,2 & + & 0,1 & + \\
\hline & 0,9 & 0,8 & 0,8 & 0,6 \\
\hline & - & 1,8 & - & 1,5 \\
\hline \multirow{3}{*}{$\begin{array}{l}\text { Ramifications } \\
\text { Racines }\end{array}$} & 0,9 & 0,6 & + & + \\
\hline & 1,1 & 0,9 & 0,3 & + \\
\hline & 0,8 & 0,7 & 1,1 & 0,9 \\
\hline
\end{tabular}

végétatifs des ramifications insérées au-dessous de la feuille alimentée en ${ }^{14} \mathrm{CO}_{2}$ draînent déjà des quantités non négligeables de métabolites marqués, alors que l'IMS des organes situés dans la partie apicale diminue considérablement. Les données correspondant à la phase de grossissement des graines sont rassemblées dans les tableaux 3 et 4 ; pour la variété Amsoy 71, les résultats relatifs aux ramifications n'ont pas été mentionnés lorsque l'IMS est inférieur à 0,1 . Les remarques suivantes peuvent être effectuées, à partir des échantillons $\mathrm{N}+$.

Dans tous les cas, exception faite du limbe et éventuellement du pétiole de la feuille ayant fixé le ${ }^{14} \mathrm{CO}_{2}$, ce sont les gousses insérées à l'aisselle de cette même

\section{TABLEAU 3}

Indice de marquage spécifique des différents organes, chez, la variété $M 13$, aux stades III et IV.

Assimilation du ${ }^{14} \mathrm{CO}_{2}$ par la $5^{e}$ feuille trifoliolée $\left(F_{5}\right) ;$ durée de la période de chasse : $6 \mathrm{~h} ;$ plantes $N+:$ plantes cultivées sur milieu complet plantes No : plantes privées d'azote à partir de la floraison; pour les ramifications, les organes végétatifs (tige et feuilles) et les organes reproducteurs sont respectivement désignés par V et $F ;+:$ IMS inférieur à 0,1 .

Relative specific activity of different plant parts in cultivar $M 13$, at developmental stages III and IV.

Fixation of ${ }^{14} \mathrm{CO}_{2}$ by the fifth trifoliate leaf $\left(F_{5}\right) ;$ chase period : $6 \mathrm{~h} ; \mathrm{N}+$ plants : plants grown on complete medium ; No plants : plants $N$ starved from flowering; for secondary axes, vegetative (stem and leaves) and reproductive organs are respectively designated $V$ and $F$; + relative specific activity $<0.1$.

\begin{tabular}{|c|c|c|c|c|c|}
\hline & & \multicolumn{2}{|c|}{ Plantes N+ } & \multicolumn{2}{|c|}{ Plantes No } \\
\hline & & Stade III & Stade IV & Stade III & Stade IV \\
\hline \multicolumn{6}{|l|}{ Axe principal: } \\
\hline \multicolumn{2}{|l|}{ Limbe $F_{5}$} & 13,1 & 11,0 & 15,2 & 14,4 \\
\hline \multicolumn{2}{|l|}{ Pétiole $\mathbf{F}_{5}$} & 3,7 & 2,9 & 2,2 & 3,9 \\
\hline \multirow{2}{*}{\multicolumn{2}{|c|}{ Feuilles $F_{1}$ à $F_{4}$}} & + & + & + & + \\
\hline & & 0,8 & 0,5 & 0,3 & 0,7 \\
\hline \multicolumn{2}{|c|}{ Epicotyle + hypocotyle } & 0,2 & 0,2 & + & 0,2 \\
\hline \multicolumn{2}{|c|}{ Gousses aisselle $F_{5}$} & 5,2 & 3,5 & 3,2 & 3,4 \\
\hline \multicolumn{2}{|c|}{ Gousses aisselle $F_{4}$} & 0,8 & 1,0 & 0,3 & 1,2 \\
\hline \multicolumn{2}{|c|}{ Gousses aisselle $F_{3}$} & 4,6 & 2,8 & 2,6 & 3,1 \\
\hline \multicolumn{2}{|c|}{ Gousses aisselle $F_{2}$} & 0,3 & 0,4 & 0,2 & 0,2 \\
\hline \multicolumn{2}{|c|}{ Gousses aisselle $F_{1}$} & 0,8 & 1,2 & 0,9 & 0,9 \\
\hline \multicolumn{6}{|l|}{ Ramifications : } \\
\hline \multirow{2}{*}{ Aisselle $F_{5}$} & $\mathrm{~V}$ & 0,1 & 0,3 & + & + \\
\hline & $\mathrm{R}$ & 2,3 & 1,1 & 2,1 & 0,7 \\
\hline \multirow{2}{*}{ Aisselle $F_{4}$} & $\mathrm{~V}$ & + & + & + & + \\
\hline & $\mathbf{R}$ & + & + & + & + \\
\hline \multirow{2}{*}{ Aisselle $F_{3}$} & $\mathrm{~V}$ & 0,4 & 0,4 & + & + \\
\hline & $\mathbf{R}$ & 3,9 & 2,3 & 0,8 & 0,7 \\
\hline \multirow{2}{*}{ Aisselle $F_{2}$} & $\mathrm{~V}$ & + & + & + & + \\
\hline & $\mathrm{R}$ & 0,2 & 0,4 & 0,1 & 0,1 \\
\hline \multirow{2}{*}{ Aisselle $F_{1}$} & $\mathrm{~V}$ & + & 0,1 & + & + \\
\hline & $\mathrm{R}$ & 1,2 & 1,3 & + & + \\
\hline \multicolumn{2}{|l|}{ Racines } & 0,5 & 0,2 & 1,9 & 0,9 \\
\hline
\end{tabular}


TABLEAU 4

Indice de marquage spécifique des divers organes, chez la variété Amsoy 71, aux stades III et IV. Assimilation du ${ }^{14} \mathrm{CO}_{2}$ par la $8^{e}$ feuille trifoliolée $\left(F_{g}\right)$. Légende identique à celle du tableau 3.

Relative specific activity of different plant parts in cultivar Amsoy 7I, at developmental stages III and IV. Fixation of ${ }^{14} \mathrm{CO}_{2}$ by the eighth trifoliate leaf (F8); same legend as in table 3.

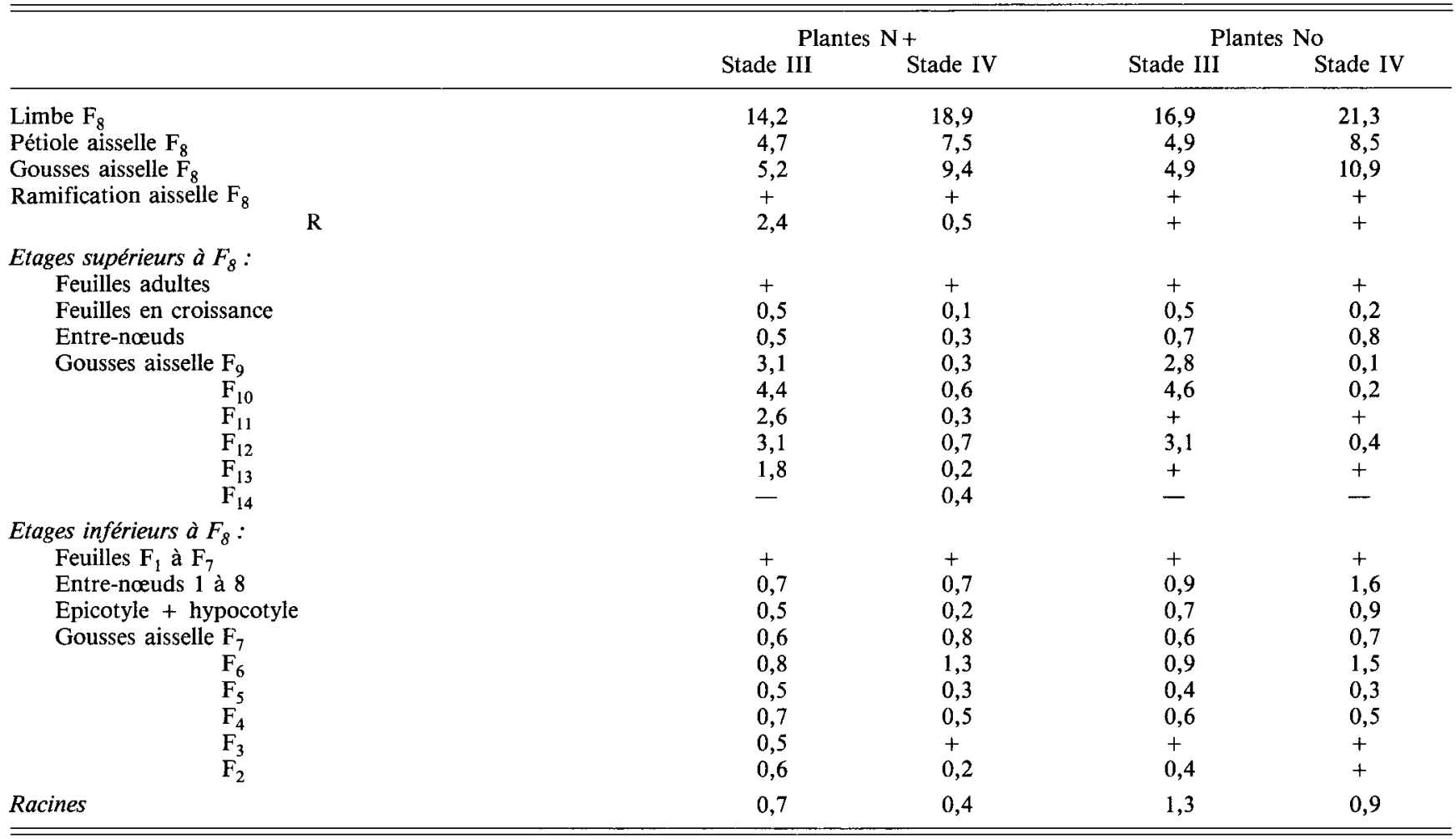

feuille qui possèdent l'IMS le plus élevé. Ceci est particulièrement net pour le cultivar Amsoy 71 et confirme que l'alimentation en carbone des fructifications est assurée en majeure partie par la feuille la plus proche. Des données similaires ont été rapportées, à propos du Soja, par divers auteurs dont STEPHENSON \& WILSON (1977) et FELlows et al. (1978). La feuille alimentée en ${ }^{14} \mathrm{CO}_{2}$ ravitaille ensuite en priorité les fruits insérés sur l'axe principal (Amsoy 71) ou sur l'axe principal et les ramifications (M 13) localisés, sur la plante, du même côté qu'elle. Ainsi, pour la variété de type déterminé où l'assimilation de ${ }^{14} \mathrm{CO}_{2}$ a été réalisée par la feuille $\mathrm{F}_{5}$, ce sont les organes qui se développent à l'aisselle des feuilles $F_{3}$ et $F_{1}$ qui sont les plus marqués, comparativement à ceux situés du côté opposé, au niveau des feuilles $\mathrm{F}_{4}$ et $\mathrm{F}_{2}$. La migration des assimilats se caractérise donc chez le Soja par une orientation longitudinale nettement préférentielle, comme il est assez fréquent de le constater chez les végétaux (BONNEMAIN, 1978).

On doit également signaler que l'âge physiologique des fruits joue un rôle important. Lorsque des gousses insérées sur le même nœud présentent des tailles différentes, celles dont le degré de développement est le plus avancé possèdent le marquage spécifique le plus fort. Ceci suggère que des déficiences trophiques pourraient expliquer, du moins partiellement, l'avortement ou le défaut de remplissage de certains organes fructifères. Ce fait mérite d'être souligné : les phénomènes d'avortement ou de coulure peuvent affecter jusqu'à 70 p. 100 des organes apparus et leur cause reste encore mal connue (BRUN \& BETTS, 1984).
En ce qui concerne les organes végétatifs, on constate que dans tous les cas le marquage des feuilles adultes est sensiblement nul ; celui des feuilles en cours de croissance, sur les ramifications chez M 13, dans les étages supérieurs de la plante chez Amsoy 71, est également faible. Durant la phase de grossissement des graines, il n'y aurait donc que des échanges réduits entre tissus assimilateurs et l'《 effet puits " des fructifications est nettement prépondérant. Enfin, on peut remarquer que l'IMS des racines est légèrement plus élevé pour Amsoy 71 que pour M13.

\section{Formes de transport}

L'étude des métabolites marqués conduit, chez le cultivar M 13, aux résultats mentionnés dans le tableau 5 ; par souci de simplification, seuls les stades II et IV ont été considérés.

L'échantillon "tissus conducteurs" regroupe le pétiole de la feuille $\mathrm{F}_{5}$, ayant incorporé le ${ }^{14} \mathrm{CO}_{2}$, et les trois entre-nœuds de l'axe principal immédiatement inférieurs. Les composés marqués présents dans ces organes possèdent une double origine. Localisés dans le phloème, ils proviennent directement de la feuille et ils représentent les formes d'exportation vers les autres parties du végétal. Ils peuvent également résulter de la transformation, au cours du métabolisme des tissus caulinaires, de substances libérées à partir des sèves. Cette seconde possibilité, selon FISHER (1970) et HOUSLEY et al. (1979), paraît avoir quantitativement une importance plus réduite. Il a donc été admis que 


\section{TABLEAU 5}

Répartition du radiocarbone dans les principaux métabolites solubles du limbe de la feuille alimentée en ${ }^{14} \mathrm{CO}$, et des tissus conducteurs. Plantes de la variété $M 13$ cultivées sur milieu complet; durée de chasse : $6 \mathrm{~h}$; résultats en \% de la radioactivité totale de la fraction soluble + : radioactivité décelée par autoradiographie mais trop faible pour être chiffrée.

Main soluble metabolites in the blade of the source leaf fed with ${ }^{14} \mathrm{CO}_{2}$ and in the conducting organs. Soybean cv. M 13, grown on complete medium; chase period: $6 \mathrm{~h}$; results expressed as \% of total labelling of the soluble fraction; + : radioactivity characterized by autoradiography but too low to be measured.

\begin{tabular}{|c|c|c|c|c|}
\hline & \multicolumn{2}{|c|}{ Tissus conducteurs } & \multicolumn{2}{|c|}{ Limbe foliaire } \\
\hline & Stade II & Stade IV & Stade II & Stade IV \\
\hline Acides aminés et amides & 31,6 & 23,0 & 24,3 & 19,9 \\
\hline Sérine & 2,7 & 0,6 & 0,5 & 0,9 \\
\hline$\alpha$-Alanine & 1,1 & 1,3 & 1,4 & 0,6 \\
\hline Aspartate & 4,6 & 2,8 & 4,8 & 4,5 \\
\hline Asparagine & 11,6 & 14,8 & 4,5 & 7,7 \\
\hline Glutamate & 5,6 & 1,3 & 3,7 & 3,5 \\
\hline Glutamine & + & + & 1,0 & 0,1 \\
\hline$\gamma$-Aminobutyrate & 3,8 & 1,2 & 4,7 & 2,0 \\
\hline Acides organiques & 18,9 & 11,4 & 48,4 & 56,0 \\
\hline Phosphoglycérate & - & - & 4,9 & 0,7 \\
\hline Glycérate & - & - & 5,9 & 1,2 \\
\hline Malonate & + & - & 1,6 & 1,2 \\
\hline Succinate & + & + & 0,7 & 1,4 \\
\hline Fumarate & + & - & 1,5 & 0,1 \\
\hline Malate & 15,8 & 6,0 & 29,8 & 44,0 \\
\hline Citrate & 3,0 & 5,3 & 1,7 & 6,5 \\
\hline Composés glucidiques & 49,5 & 65,6 & 27,3 & 24,1 \\
\hline Oses phosphate & + & + & 3,1 & 1,4 \\
\hline Glucose & 14,2 & 8,4 & 6,2 & 7,7 \\
\hline Fructose & 12,7 & 7,5 & 4,4 & 7,2 \\
\hline Saccharose & 20,7 & 49,6 & 8,5 & 5,8 \\
\hline Maltose & 1,8 & + & 1,9 & 2,0 \\
\hline
\end{tabular}

la nature des produits solubles marqués détectés dans les tissus conducteurs constituait un fidèle reflet des échanges s'effectuant entre organes.

L'analyse de la fraction cationique permet de souligner l'importance de l'asparagine et, à un degré moindre, celle de l'aspartate et du glutamate dans les transferts d'azote ; on peut également remarquer la participation de la sérine, vraisemblablement issue du métabolisme photorespiratoire (LATCHÉ et al., 1978). Aucune incorporation de radiocarbone n'a été décelée dans les uréides glyoxyliques, allantoïne et acide allantoïque. Les uréides sont les constituants azotés majeurs des sèves chez le soja nodulé ; en absence de nodulation, leur concentration est très faible, voire nulle, et le rôle de l'asparagine devient alors prépondérant (PATE, 1980). Le saccharose est le principal composé marqué dans la fraction neutre et il constitue la forme essentielle de migration du carbone. Un résultat similaire a été notamment reporté par FISHER (1970), HOUSLEY et al. (1979) et KOCH \& SCHRADER (1984). Enfin, de façon générale, la présence d'acides organiques dans le phloème est assez peu fréquente (BONNEMAIN, 1978). Toutefois, ceux-ci pourraient participer aux transferts de carbone chez divers végétaux, dont la rhubarbe, Robinia pseudoacacia (L.), Ricinus communis (L.), Cucurbita ficifolia (Bouché) et Larix decidua (L.); les acides malique, fumarique, succinique, citrique, oxalique, quinique et shikimique sont le plus souvent impliqués dans ces processus (ZIEGLER, 1975). Plus récemment, la caractérisation d'acides organiques, et en particulier de malate et de citrate, dans les sèves de soja a été reportée par LAYZELL \& LA RUE (1982), ce qui est en accord avec les résultats acquis au cours de ce travail.
L'examen des données en fonction du stade de développement montre, du stade II au stade IV, une augmentation de la radioactivité des composés glucidiques, alors que celle des acides organiques et des substances azotées solubles diminue. Cette dernière constatation est à rapprocher de l'évolution de l'activité de la nitrate réductase foliaire, plus faible dans les tissus âgés (LATCHÉ et al., 1986), ce qui suppose que des quantités moindres d'azote soient alors disponibles pour la biosynthèse d'aminoacides exportables. On remarque par ailleurs que la diversité des formes de migration s'atténue avec l'âge de la plante : au stade IV, asparagine et saccharose renferment 64,5 p. 100 de l'ensemble du radiocarbone de la fraction soluble, contre seulement 32,3 p. 100 au stade II.

L'analyse du limbe de la feuille $F_{5}$, ayant fixé le ${ }^{14} \mathrm{CO}_{2}$, fait enfin ressortir de notables différences comparativement aux résultats acquis à partir des tissus conducteurs. Le limbe se caractérise en effet par un marquage nettement plus élevé des acides organiques, tandis que les glucides et les constituants azotés présentent une radioactivité plus faible. En outre, la répartition du radiocarbone entre les divers composés varie : ainsi, le malate est de loin la substance la plus marquée et la prépondérance de l'asparagine et du saccharose, respectivement dans les fractions cationique et neutre, n'est plus observée. Ceci confirme que tous les métabolites solubles élaborés au niveau du limbe ne peuvent jouer un rôle identique dans les transferts entre la feuille et les autres organes de la plante.

Par rapport à la variété $\mathrm{M} 13$, les données obtenues pour le cultivar Amsoy 71 conduisent à des conclusions similaires ; toutefois, quelques variations de fai- 
ble amplitude ont été notées. Au stade IV par exemple, le marquage du saccharose dans les tissus conducteurs est encore plus important et, dans le limbe, le glutamate, la glutamine et le citrate incorporent proportionnellement davantage de ${ }^{14} \mathrm{C}$. Ces faits suggèrent l'existence de différences métaboliques mineures, en fonction de la variété de soja envisagée, ce que confirment les travaux de HouSLEY et al. (1979) et de LAYZELL \& LA RUE (1982) menés sur les cultivars Wells et Wilkin.

\section{B. Etude des répercussions de la privation d'azote}

\section{Effets de la carence sur le développement du soja}

De façon générale, et bien que l'ensemble de la plante soit affecté, la carence en azote influe surtout sur le développèment des ramifications et elle se répercute davantage sur les organes reproducteurs que sur les organes végétatifs. Les résultats acquis à partir de la variété $M 13$, regroupés dans le tableau 6 , illustrent ce phénomène.

Après 14 jours de carence (stade III), la perte de masse des gousses est de 35 p. 100 au niveau de l'axe principal, contre 41,4 p. 100 sur les ramifications ; au stade IV, ces valeurs atteignent 24 p. 100 et 68 p. 100. Ces différences proviennent essentiellement de la diminution du nombre de fructifications ; elle affecte dans de plus fortes proportions les gousses renfermant 1 ou 2 grains (respectivement -92 p. 100 et -53 p. 100) que les gousses mieux remplies ( -27 p. 100). Ce résultat est à rapprocher des observations de BoUNIOLS et al. (1981), qui montrent que la fréquence d'occupation des loges carpellaires par des graines est plus élevée chez des plantes ayant subi un déficit d'alimentation en azote. En ce qui concerne les organes végétatifs, on note d'abord une réduction du nombre et de la taille des feuilles portées par les ramifications ; en outre, toutes les feuilles qui étaient en voie de formation au moment de la floraison (début de la carence), ou qui se sont formées par la suite, présen- tent des symptômes de chlorose ; au stade IV, leurs teneurs en chlorophylle sont 4 à 8 fois inférieures à celles des échantillons témoins normalement alimentés (Grima-PETTENATI, 1985) ; enfin, la sénescence et la chute des feuilles âgées, correspondant aux étages inférieurs, est accélérée. La masse des racines est en revanche un peu plus importante $(+9$ p. 100) chez les plantes No au stade III ; cependant, cette accélération du développement racinaire ne se poursuit pas lorsque le traitement se prolonge (stade IV).

\section{Transferts des photosynthétats chez les plantes carencées}

La comparaison des figures 1 et 2 montre que la privation d'azote provoque une réduction de l'exportation des assimilats élaborés dans le limbe de la feuille alimentée en ${ }^{14} \mathrm{CO}_{2}$. Après $6 \mathrm{~h}$ de chasse, cette réduction chez la variété $\mathrm{M} 13$ atteint 47 p. 100 au stade III et 53,5 p. 100 au stade IV; pour le cultivar

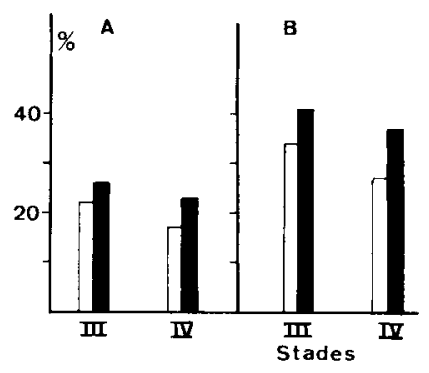

Figure 2

Radioactivité totale des métabolites exportés à partir de la feuille alimentée en ${ }^{14} \mathrm{CO}_{2}$, après une période de chasse de $6 h(A)$ ou $24 h(B)$.

Résultats exprimés en \% de la radioactivité fixée.

$\square$ variété Amsoy $71 ; \quad$ variété $M 13$; plantes cultivées sur milieu dépourvu d'azote, à partir du début de floraison.

Total labelling of metabolites exported from the source leaf fed with ${ }^{14} \mathrm{CO}_{2}$, after a $6 \mathrm{~h}(\mathrm{~A})$ or $24 \mathrm{~h}(\mathrm{~B})$ chase period. Results expressed as $\%$ of fixed ${ }^{14} \mathrm{C}$.

$\square$ cultivar Amsoy $71 ; \square$ cultivar M 13 ; plants $N$-starved from flowering.

\section{TABLEAU 6}

Masse des différents organes, en fonction du stade de développement et de la composition du milieu de culture.

Plantes $N+$ : plantes cultivées sur milieu complet; plantes No: plantes privées d'azote depuis 14 jours (stade III) ou 21 jours (stade II) colonne $a:$ masse en $g$ de matière sèche; colonne $b: \%$ de la masse totale de la plante.

Dry weight of different organs, in relation to plant development and nutritive medium composition. $N+$ plants : plants grown on complete medium ; No plants : plants $N$-starved during 14 (stage III) or 21 days (stage IV); a : dry matter ; $b: \%$ of total plant dry matter.

\begin{tabular}{|c|c|c|c|c|c|c|c|c|}
\hline & \multicolumn{4}{|c|}{ Plantes N+ } & \multicolumn{4}{|c|}{ Plantes No } \\
\hline & \multicolumn{2}{|c|}{ Stade III } & \multicolumn{2}{|c|}{ Stade IV } & \multicolumn{2}{|c|}{ Stade III } & \multicolumn{2}{|c|}{ Stade IV } \\
\hline & a & b & a & b & $\mathrm{a}$ & $\mathrm{b}$ & a & b \\
\hline \multicolumn{9}{|l|}{ Axe principal: } \\
\hline feuilles & 5,1 & 21,5 & 5,6 & 13,1 & $5,0^{\bullet}$ & 24,3 & 3,3 & 15,5 \\
\hline tige & 3,9 & 16,5 & 5,7 & 13,4 & 4,2 & 20,4 & 4,3 & 20,2 \\
\hline organes reproducteurs & 2,1 & 8,9 & 4,6 & 10,8 & 1,4 & 6,8 & 2,3 & 10,8 \\
\hline \multicolumn{9}{|l|}{ Ramifications : } \\
\hline organes végétatifs & 6,8 & 28,7 & 12,9 & 30,2 & 4,9 & 23,8 & 4,2 & 19,7 \\
\hline organes reproducteurs & 2,4 & 10,1 & 9,1 & 21,3 & 1,4 & 6,8 & 2,9 & 13,6 \\
\hline Racines & 3,4 & 14,3 & 4,8 & 11,2 & 3,7 & 17,9 & 4,3 & 20,2 \\
\hline Total & 23,7 & & 42,7 & & 20,6 & & 21,3 & \\
\hline
\end{tabular}


Amsoy 71 , les résultats sont sensiblement identiques au stade III $(-46$ p. 100) et un peu plus élevés au stade suivant ( $-57,3$ p. 100). Toutefois, les différences observées entre les échantillons $\mathrm{N}+$ et No s'atténuent au terme d'une période de chasse de $24 \mathrm{~h}$ : -29 et -35 p. 100 chez M 13 contre -28 et - 38 p. 100 chez Amsoy 71, aux stades III et IV respectivement. Les perturbations consécutives du stress azoté s'expliquent donc à la fois par un ralentissement de la vitesse des échanges et par une baisse du volume global de ces derniers.

Les principales variations entre plantes témoins et carencées dans la répartition du radiocarbone exporté sont illustrées dans la figure 3 . La déficience en azote se traduit d'abord par une forte augmentation du transfert des assimilats vers les racines ; elle est plus marquée chez la variété M 13 et, bien que se manifestant plus intensément au stade III, elle persiste dans les végétaux plus âgés. Une observation similaire a été effectuée à propos du blé par CHAMPIGNY et al. (1983). On note également, chez les plantes carencées, une distribution plus faible du radiocarbone dans les ramifications. Cet ensemble de données est à rapprocher des modifications morphologiques constatées dans les échantillons No. Enfin, au terme de $6 \mathrm{~h}$ de chasse, une accumulation de ${ }^{14} \mathrm{C}$ apparaît dans les tissus conducteurs, particulièrement prononcée après 21 jours de carence ; quoiqu'elle soit moins visible au bout de $24 \mathrm{~h}$ de chasse, elle prouve le ralentissement de la vitesse de circulation des assimilats.

Le calcul des indices de marquage spécifique (tabl. 3 et 4) confirme ces différentes constatations. Dans les échantillons No, le limbe de la feuille ayant fixé le ${ }^{14} \mathrm{CO}_{2}$ présente un IMS plus élevé, résultant de la diminution de l'exportation des photosynthétats ; de même, l'IMS des racines et celui des entre-nœuds de la partie inférieure de la tige (au stade III et surtout au stade IV) ont une valeur plus forte que pour les plantes $\mathrm{N}+$.

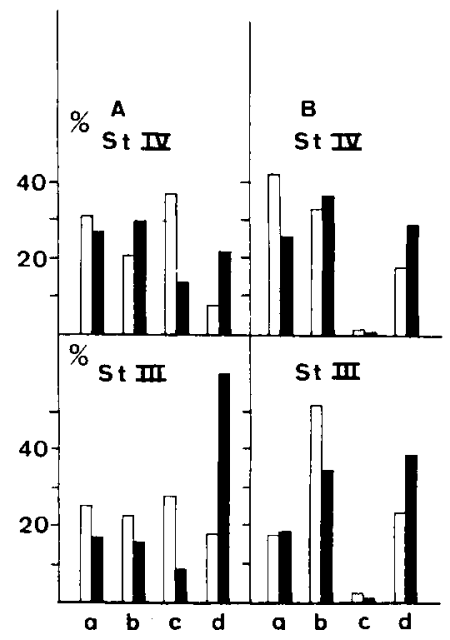

Figure 3

Répartition de la radioactivité, en \%, chez la variété $M 13(A)$ et la variété Amsoy 71 (B) cultivées sur milieu complet $\square$ ou sur milieu dépourvu d'azote

$a$ : organes insérés à l'aisselle de la feuille alimentée en ${ }^{14} \mathrm{CO}_{2}$.

$b:$ tige principale + organes insérés sur la tige principale.

$c$ : ramifications.

$d$ : racines.

Distribution of labelled carbon (\%) in cultivars $M 13$ (A) and Amsoy 71 (B) plants grown on complete medium $\square$ or $N$-starved from flowering

$a$ : organs in the axil of the labelled leaf.

$b:$ main stem + leaves or pods located on the main stem.

$c$ : secondary axes.

$d:$ roots

\section{Principaux métabolites marqués}

Chez les plantes carencées, la radioactivité des composés cationiques, et plus spécialement celle de l'asparagine et de l'aspartate, subit une baisse sensible. Parallèlement à ce résultat logique consécutif à la privation d'azote, on note que le marquage des acides organiques diminue, surtout dans le limbe (tabl. 7).

\section{TABLEAU 7}

Principaux métabolites solubles marqués dans les tissus conducteurs et dans le limbe de la feuille alimentée en ${ }^{14} \mathrm{CO}_{2}$, chez les plantes de la variete $M 13$ soumises a une privaton d'asole.

Légende identique à celle du tableau 5.

$\mathrm{N}$-starved plants cv. M 13 : main soluble labelled metabolites in the conducting organs and in the blade of the source leaf fed with ${ }^{14} \mathrm{CO}_{2}$ Same legend as in table 5.

\begin{tabular}{|c|c|c|c|c|}
\hline & Tissus & cteurs & Lim & aire \\
\hline & Stade III & Stade IV & Stade III & Stade IV \\
\hline Acides aminés et amides & 10,9 & 2,7 & 7,7 & 5,7 \\
\hline$\alpha$-Alanine & + & - & 1,3 & + \\
\hline Aspartate & 2,5 & 0,5 & 0,5 & + \\
\hline Asparagine & 5,4 & 1,0 & 0,5 & + \\
\hline Glutamate & 2,9 & 0,6 & 3,0 & 2,0 \\
\hline$\gamma$-Aminobutyrate & + & + & 2,4 & 3,5 \\
\hline Acides organiques & 11,1 & 6,1 & 26,2 & 8,9 \\
\hline Phosphoglycérate & + & + & 1,1 & 0,5 \\
\hline Glycérate & + & + & 1,1 & 0,7 \\
\hline Succinate & + & + & 2,3 & 0,6 \\
\hline Malate & 6,0 & 3,1 & 18,1 & 4,9 \\
\hline Citrate & 3,1 & 2,0 & 1,8 & 0,8 \\
\hline Composés glucidiques & 78,0 & 91,2 & 66,1 & 85,4 \\
\hline Oses phosphate & + & + & 0,9 & 5,8 \\
\hline Glucose & 5,8 & 6,1 & 17,8 & 9,4 \\
\hline Fructose & 4,9 & 5,1 & 13,8 & 10,7 \\
\hline Saccharose & 61,8 & 79,9 & 30,5 & 54,8 \\
\hline
\end{tabular}


La part prise par la fraction glucidique au sein de la fraction soluble devient donc prépondérante dans les échantillons No. Le saccharose est de loin le composé le plus marqué et, chez la variété M 13 au stade IV, il renferme près de 80 p. 100 de la totalité du ${ }^{14} \mathrm{C}$ retrouvé dans les tissus conducteurs. La même constatations a été effectuée pour la variété Amsoy 71, bien que la prédominance du saccharose soit moins nette et que glucose et fructose présentent une radioactivité non négligeable.

\section{CONCLUSION}

Ce travail a permis de dégager les différences existant, au niveau du transfert des photosynthétats, entre variétés de soja de type déterminé ou indéterminé. Chez la variété $M 13$, dont la tige présente une croissance plus réduite, les feuilles de niveau moyen alimentent en composés organiques à la fois les organes reproducteurs insérés sur l'axe principal et ceux situés sur les ramifications. En revanche pour la variété Amsoy 71, de type indéterminé, les exportations vers les parties apicales se poursuivent plus longtemps au cours du cycle de développement et les ramifications présentent une autonomie assez poussée, ne recevant que très peu d'assimilats provenant des feuilles portées par la tige principale. On doit en outre signaler, chez les deux cultivars, l'orientation longitudinale stricte des migrations. Ces différents caractères se retrouvent chez des plants de soja nodulés, bien que l'effet «puits » des nodosités réduise la proportion de carbone parvenant aux gousses, au moins durant les premières étapes de la phase de grossissement des graines (J. CALMÉs, G. Viala et J.C. LATCHÉ, résultats non publiés ; PaUl \& PlanCHON, 1983).
La privation d'azote provoque essentiellement une diminution de l'exportation des assimilats vers les ramifications, alors que les racines, dont le développement est accéléré, sont mieux alimentées. En outre, le marquage plus élevé des organes conducteurs plaide en faveur d'un ralentissement de la vitesse des échanges. Les explications physiologiques de ces différents effets paraissent complexes. Les végétaux carencés présentent une baisse de photosynthèse nette, dont les causes sont multiples (GRIMA-PETTENATI et al., 1986), et l'intensité respiratoire est également diminuée (GRIMA-PETTENATI, 1985). Il en résulte une réduction de la biosynthèse de molécules carbonées organiques, mais aussi d'ATP, sans doute préjudiciable aux mécanismes de transfert actifs. Par ailleurs, l'importance du saccharose parmi les formes de transport a été soulignée ; selon HUBER (1984), des déficiences dans l'alimentation minérale sont susceptibles de perturber le métabolisme de ce diholoside, ce qui se répercute sur le volume des migrations. Enfin, les plantes No ayant un rendement en graines nettement plus faible, l'effet " puits » des fructifications ne peut se manifester normalement. Ainsi, GRIMA-PETTENATI (1985) a montré que les échanges de photosynthétats sont moins modifiés par la privation d'azote chez des plants de soja privés d'organes fructifères. Ces faits pourraient être reliés à des phénomènes hormonaux, une accumulation d'acide abscissique ayant été signalée chez le tournesol (GOLDBACH et al., 1975) et le cotonnier (RADIN \& ACKERSON, 1981) déficients en azote. Des travaux ultérieurs sont nécessaires pour tester la validité de ces diverses hypothèses et déterminer l'importance respective des paramètres envisagés.

Reçu le 20 octobre 1986. Accepté le 10 mars 1987.

\section{RÉFÉRENCES BIBLIOGRAPHIQUES}

Andrews A. K., Svec L. V., 1975. Photosynthetic activity of soybean pods at different growth stages compared to leaves. Can. J. Plant Sci., 55, 501-505.

Bonnemain J. L., 1978. Transport et distribution des produits de la photosynthèse. In : C. Costes, Photosynthèse et production végétale, Gauthier-Villars, 171-194.

Bouniols A., Puech J., Hernandez M., Salvy J., Mondies M., 1981. Effet d'une privation d'azote à différents stades du développement du Soja (Glycine $\max$ L. Merrill) : conséquences sur la mise à fleurs, sur la production fructifère et sur la teneur en protéines des graines récoltées. C.R. Acad. Sci., Paris, Série III, 293, 97-102.

Brun W. A., Betts K. J., 1984. Source-sink relations of abscissing and non abscissing soybean flowers. Plant Physiol, 75, 187-191.

Calmés J., Viala G., Gelfi N., Błanchet R., 1985. Influence d'un déficit hydrique sur trois variétés de Soja : effet sur la protéogenèse des graines. Agronomie, 5, 169-176.

Champigny M. L., Bismuth E., Murty A., 1983. The effect of N-or P-deficiencies on processes related to carbon metabolism in Triticum aestivum. In : N. Metzner, Photosynthesis and plant productivity, W. V. MbH, Stuttgart, 116-121.

Cure J. D., Patterson R. P., Raper C. D., Jr., Jackson W. A., 1982. Assimilate distribution in soybeans as affected by photoperiod during seed development. Crop Sci., 22, 1245-1250.

Fehr W. R., Caviness C. E., Burmood D. T., Pennington J. S., 1971. Stage of development descriptions for soybean, Glycine max (L.) Merrill, Crop. Sci., 11, 929-931.
Fellows R. J., Egli D. B., Leggett J. E., 1978. A pod leakage technique for phloem translocation studies in soybean (Glycine max [L.] Merr.). Plant Physiol., 62, 812-814.

Fisher D. B., 1970. Kinetics of C-14 translocation in soybean. I. Kinetics in the stem. Plant Physiol., 45, 107-113.

Goldbach E., Goldbach H., Wagner H., Michael G., 1975. Influence of $\mathrm{N}$-deficiency on the abscissis acid content of sunflower plants. Physiol. Plant., 34, 138-140.

Grima-Pettenati J., 1985. Effets d'une privation d'azote sur la nutrition carbonée et la dégradation des protéines foliaires chez le Soja. Thèse Doct. $3^{\mathrm{e}}$ cycle, Université Paul Sabatier, Toulouse, $116 \mathrm{p}$.

Grima-Pettenati J., Piquemal M., Latché J. C., 1986. Effets d'une privation d'azote sur le métabolisme photosynthétique chez le soja. Physiol. vég., 24, 569-580.

Heindl J. C., Brun W. A., 1983. Light and shade effects on abscission and ${ }^{14} \mathrm{C}$-photoassimilate partitioning among reproductive structures in soybean. Plant Physiol., 73, 434-439.

Housley T. L., Schrader L. E., Miller M., Setter T. L., 1979. Partitioning of ${ }^{14} \mathrm{C}$-photosynthate, and long distance translocation of amino acids in preflowering and flowering, nodulated and non nodulated soybeans. Plant Physiol., 64, 94-98.

Huber S. C., 1984. Biochemical basis for effects of K-deficiency on assimilate export rate and accumulation of soluble sugars in soybean leaves. Plant Physiol., 76, 424-430. 
Huber S. C., Rogers H., Israel D. W., 1984a. Effects of $\mathrm{CO}_{2}$ enrichment on photosynthesis and photosynthate partitioning in soybean (Glycine max) leaves. Physiol. Plant., 62, 95-101.

Huber S. C., Rogers H. H., Mowry F. L., 1984b. Effects of water stress on photosynthesis and carbon partitioning in soybean (Glycine max [L.] Merr.) plants grown in the field at different $\mathrm{CO}_{2}$ levels. Plant Physiol., 76, 244-249.

Koch K. E., Schrader L. E., 1984. ${ }^{14} \mathrm{C}$-Photosynthate partitioning and translocation in soybeans during reproductive development. Plant Physiol., 75, 1040-1043.

Kpodar M. P., Piquemal M., Calmés J., Latché J. C., 1978. Relations entre nutrition azotée et métabolisme photorespiratoire chez une plante à oxalate, Fagopyrum esculentum M. Physiol. vég., 16, 117-130.

Latché J. C., Viala G., Calmès J., Cavalié G., 1978. Etude comparative du métabolisme photorespiratoire chez différentes variétés de Soja. Ann. Amélior. Plantes, 28, 77-87.

Latché J. C., Bailly-Fenech G., Grima-Pettenati J., Cavalié G., 1986. Etude comparative du métabolisme photosynthétique des feuilles et des gousses de soja, Glycine max (L.) Merrill. Can. J. Bot., 64, 1542-1548.

Layzell D. B., La Rue T. A., 1982. Modeling C and N transport to developing soybean fruits. Plant Physiol., 70, 1290-1298.

Louguet P., 1978. Les stomates : caractères principaux et mécanismes de leur mouvement. In : C. Costes, Photosynthèse et production végétale, Gauthier-Villars, 195-217.

Obaton M., 1980. Activité nitrate réductase et nitrogénase chez le soja en relation avec la photosynthèse et les facteurs de l'environnement. Le Sélectionneur Français, 28, 56-60.

Oliker M., Mayer A. M., Poljakoff-Mayber A., 1978. Changes in weight, nitrogen accumulation, respiration and photosynthesis during growth and development of seeds and pods of Phaseolus vulgaris. Amer. J. Bot., 65, 366-371.

Pate J. S., 1980. Transport and partitioning in nitrogenous solutes. Annu. Rev. Plant Physiol., 31, 313-340.

Paul M. H., Planchon C., 1983. Transport et distribution des assimilats pendant la phase floraison-grossissement des graines chez un soja de type déterminé (cv M 13). C. R. Acad. Sci., Paris, Série III, 297, 405-410.

Radin J. W., Ackerson R. C., 1981. Water relations of cotton plants under nitrogen deficiency. III. Stomatal conductance, photosynthesis, and abscissic acid accumulation during drought. Plant Physiol., 67, 115-119.

Sambo E. Y., Moorby J., Milthorpe F. L., 1977. Photosynthesis and respiration of developing soybean pods. Austr. J. Plant Physiol., 4, 713-721.

Seddigh M., Joliff G. D., 1984. Effects of night temperature on dry matter partitioning and seed growth of indeterminate field-grown soybean. Crop. Sci., 24, 704-710.

Stephenson R. A., Wilson C. T., 1977. Patterns of assimilate distribution in soybeans at maturing. Austr. J. Agric. Res., 28, 203-209.

Ziegler H., 1975. Nature of substances in phioem. In : M. H. Zimmermann et J. A. Milburn, Transport in plants, vol. I, SpringerVerlag, Berlin, Heidelberg, New York. 